\title{
Autonomous Driving in a Time-Varying Environment ${ }^{*}$
}

\author{
Toby Myers, Ljubo Vlacic \\ School of Microelectronic Engineering \\ Griffith University \\ Nathan Campus, Brisbane, Qld 4111, Australia \\ Toby.Myers@student.gu.edu.au,L.Vlacic@griffith.edu.au
}

\author{
Tony Noel, Michel Parent \\ IMARA Research Team \\ INRIA Rocquencourt \\ BP 105, 78153 Le Chesney, France \\ \{Tony.Noel, Michel.Parent\}@inria.fr
}

\begin{abstract}
Robot motion is a field of continuing and active research that has recorded a number of achievements in the last decade, but research appears to be becoming stagnant in key areas. Current researchers limit the success of their work by using sensors with limited features capable of operating in static environments with known static obstacles and not considering implementation on non-holonomic vehicles. These simplifications of the task of dynamic obstacle avoidance greatly reduce the possible applications of current robot motion algorithms in areas such as Autonomous Driving. This paper deals with algorithms for on-the-ny avoidance of dynamic obstacle by presenting a new approach, the TimeVarying Dynamic Window algorithm capable of operating at high speeds on a non-holonomic vehicle in an environment that changes over time.
\end{abstract}

Index Terms - Intelligent Vehicles, Autonomous Driving, Dynamic Window, Dynamic Obstacles

\section{INTRODUCTION}

Robot motion, specifically in the area of dynamic obstacle avoidance represents an exciting area of research. In order for success to be achieved in this field a number of areas of research must be integrated including sensor design, data fusion, map building, localization, off-line optimized path planning methods and reactive high-speed obstacle avoidance methods.

Current sensor technology available to obstacle avoidance includes GPS, ladar (laser detection and ranging) in both two and three dimensions, sonar, microwave radar and $\mathrm{CCD}$ cameras. In addition to the features specific to each of these sensor technologies, the factors should be considered when choosing a sensor setup for a platform include cost, computational complexity of processing, response time, field of vision, resolution, range of detection, operation in two/three dimensional plane and the effect of adverse weather conditions.

Most research to date in obstacle avoidance has made use of ultrasonic sensors or sonar due to its low cost and simplicity of use. Unfortunately these advantages are far outweighed by a very small range of detection (3m at $40 \mathrm{kHz})$, a poor angular accuracy $\left(+/-5^{\circ}\right)$ and total failure in adverse weather conditions such as strong winds or platforms operating at high speeds [1]. Furthermore, problems with crosstalk can cause a sonar sensor with a $60 \mathrm{~ms}$ response operating in a ring configuration can have a response time of the entire ring of $300 \mathrm{~ms}$ which is unacceptable for real-time operation. These problems render the algorithms created specifically for this type of sensor useless for all but the most trivial applications in which the platform operates in an indoor static environment at low speeds.

Another sensor to gain popularity more recently has been the ladar sensor. Its large range of detection, fast response time, and low complexity of processing [2] make it ideal for outdoor high speed applications such as autonomous driving. Its main drawback is that in adverse weather conditions rain, snow, and dirt can be perceived as false objects.

Also different types of sensors are being continually developed. A recently developed prototype intelligent motion estimation sensor, implemented using Field Programmable Gate Array (FPGA) technology and a combination of visual information (from a camera) together with range information (from a laser range scanner) used to give an estimate of the motion in the environment, is a great step forward towards solving the motion estimation problem [3]. However, it is yet to become commercially available.

A good compromise at this research stage would be to use data fusion to combine information from a ladar sensor with short-wave radar, which is not susceptible to adverse weather conditions, thereby creating a failsafe sensor setup. The only drawback to this configuration is the current high cost of the short-wave radar technology.

Another limitation present in current research is most algorithms have been designed to operate in an static timeinvariant environment using holonomic vehicles. Adopting terminology from Hwang and Ahuja [4] the following definitions will be used for defining an environment that a obstacle avoidance method is designed for. A Static environment is the case in which all obstacle information is know prior to the planning of the method beginning whereas in a Dynamic environment the information of the environment becomes known over time by way of information provided by sensors. An environment can also be classified as Time Varient/Invarient depending on whether the position of obstacles changes or doesn't change over time respectively. A Holonomic vehicle is a vehicle capable of unconstrained motion such as turning on its axis and near instant acceleration within a specified (normally low) velocity range. From these definitions it is obvious that researchers have avoided the complexities of the obstacle avoidance problem by making unfeasible assumptions and thereby limiting the possible applications of their research.

This is exemplified by the fact that most researchers believe that by making an obstacle avoidance method suitable for a static time-invariant environment it is only a matter of increasing processing power and applying small

\footnotetext{
- This work was supported in part by the French Government through the IMARA research unit of INRLA Rocquencourt. Support was also provided by Griffith University, Australia and its Institute for Intelligent and Integrated Systems.
} 
modifications to achieve the same success in a dynamic time-varying environment. This is strongly refuted by Kohout [5] and has lead to the creation of the Time-varying Dynamic Window Approach.

The organization of this paper is as follows. In Section II, areas of related work will be discussed. Section III summarizes the Dynamic window approach. Section IV gives a brief explanation of the Velocity Obstacle approach. Our proposed time-varying reactive method based on a combination of the Dynamic Window approach and the Velocity Obstacle approach is detailed in Section V. In Section VI, experimental results obtained from both the simulated environment and on-road experimenting with an autonomous vehicle are presented. Finally, the conclusions can be found in Section VII including comments on the acceptance of the technology derived from the entirety of the experiments so far performed by driverless vehicles.

\section{RELATED WORK}

The separation of obstacle avoidance into different methods is a very controversial subject in which there are many differing opinions. Most agree that there are two approaches, global and local (though not always with these titles). This however is where the agreement ends and due to different authors differing definitions it is common to find methods classified as global by one author and local by another.

For the purposes of this paper the following definitions of global and local will be used. Global methods operate in a static environment by computing off-line an optimised path from start to finish that avoids all known static obstacles. This approach cannot deal with incomplete or inaccurate information or a time-varying environment and the complexity of this approach means that replanning is too computationally expensive.

Local methods use only a small fraction of the world space and operate in real-time in a dynamic, time-varying environment. They have the disadvantage of not being able to produce optimal solution and can get trapped in local minima (such as a large U shaped obstacle) [4].

Using this definition of Local methods there are two distinct types of methods that fit this category; Local Path Planners and reactive methods. Local Path Planning methods map out the entire path (made under the assumption of static obstacles) and then make adjustments while following the planned path. The most well-known local path planning method is the potential field approach which has been implemented using a wide variety of different methods including a method to remove local minima using harmonic functions [4].

Reactive methods make angular and translation velocity commands based upon information processed from current sensory data. Current Reactive methods include the curvature velocity method, the Dynamic Window approach [6], Velocity Obstacles approach [7], Vector Field Histograms, Polar Object Chart method, and Fuzzy Logic.

The combination of Local and Global methods into an integrated system is called a Hybrid method. These methods are designed to combine the advantages of both methods and remove the disadvantages of each operating singularly. Most hybrid methods operate by using a global path planner to provide sub-points along an optimised path that are then used as goal points for a local method.

\section{DYNAMIC WINDOW ALgORITHM}

The dynamic window approach [6] is an obstacleavoidance method that is capable of operating in a timeinvariant environment dynamically updated by information provided by a sensor. The algorithm is designed to use a non-holonomic vehicle that takes into account the kinematics and dynamics of syncro-drive robots. The kinematics of the vehicle is considered by searching the velocity space $(\nu, \omega)$ consisting of the translational velocities $v$ and angular velocities $\omega$ that are achievable by the robot.

The dynamic window approach is implemented in four steps each of which involves a further reduction of the available velocities until the best possible translational and angular velocity has been chosen. The first step restricts the velocity space to the achievable velocities, which are the set of translational and angular velocities achievable with the kinematic constraints of the vehicle.

This set of achievable velocities is then reduced to those velocities that can safely avoid obstacles near the vehicle. The resultant set of velocities is called the admissible velocities. For the purposes of this method obstacles are considered avoided if the robot can stop without a collision. This definition is far from ideal and does not calculate future points of collision as is necessary to successfully avoid dynamic obstacles.

The third step of the method is to create a dynamic window of the admissible velocities in which only velocities, which can be reached within a set time frame and within the acceleration constraints of the vehicle, remain. This is normally a rectangular window centred on the current velocity of the vehicle and extended according to the vehicles acceleration capabilities.

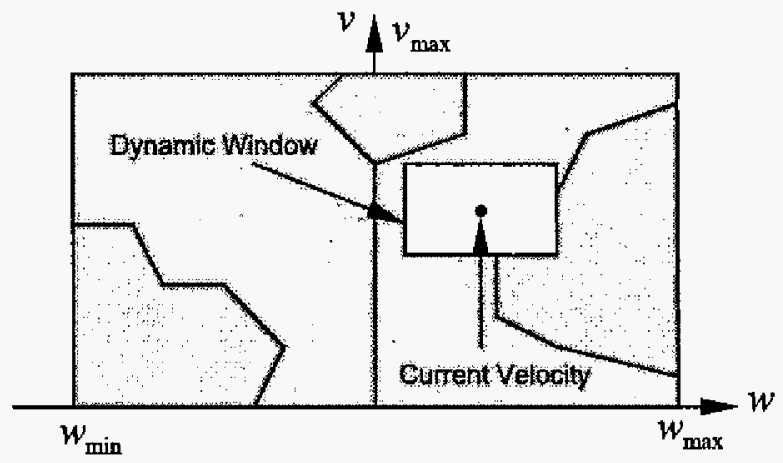

Fig. 1 Dynamic Window Search Space [6]

The fourth and final step of the dynamic window approach is to search the dynamic window using a cost function to find the best translational and angular velocity based on a set of heuristics. The heuristics of the cost function favour velocities in the direction of the goal, angle $(v, \omega)$, that maintain a large distance from obstacles, dist $(v, \omega)$, and that operate at faster speeds, velocity $(v, \omega)$. This is all incorporated into a weighted function that allows 
the relative importance of each of the behaviors to be modified,

$$
G(v, w)=\sigma(\alpha \cdot \text { angle }(v, w)+\beta \cdot \operatorname{dist}(v, w)+\gamma \cdot v \text { elocity }(v, w)) . .(1)
$$

which is then computed over the discretized set of translational and angular velocities located in the dynamic window.

\section{VELOCITY OBSTACLE ALGORITHM}

The Velocity Obstacle approach [7] unlike the majority of other obstacle avoidance methods is capable of operating in a Time-Varying environment as it has been specifically designed to avoid moving obstacles.

In order to calculate the Velocity Obstacle (VO) consider two circular objects, $A$ and $B$, at time $t_{0}$, with velocities $v_{A}$ and $v_{B}$ where $A$ represents the robot and $B$ represents a moving obstacle, Fig. 2 . The first step is to reduce the robot circle, $A$, to a single point, $\hat{A}$ and to enlarge the object circle, $B$ by the radius of $A$ to $\hat{B}$. A Collision Cone, $C C_{A, B}$ is then created which is the set of colliding relative velocities between $\hat{A}$ and $\hat{B}$ and is defined as:

$$
C C_{A, B}=\left\{v_{A, B}: \lambda_{A, B} \cap \hat{B} \neq 0\right\}
$$

where $v_{A, B}$ is the relative velocity of $\hat{A}$ with respect to $\hat{B}$, $v_{A, B}=v_{A}-v_{B}$, and $\lambda_{A, B}$ is the supporting line of $v_{A, B}$. Any relative velocity within $C C_{A, B}$ will cause $\mathrm{A}$ and $\mathrm{B}$ to collide.

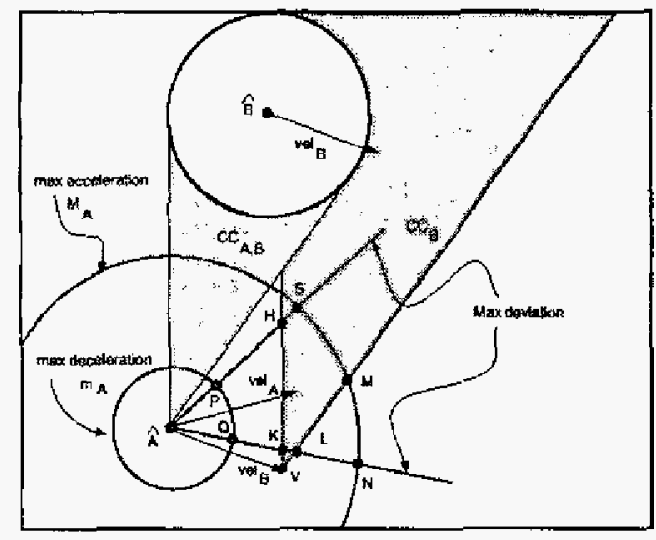

Fig. 2 Construction of a Velocity Obstacle [7]

In order to avoid multiple obstacles, absolute velocities must be used by adding the velocity of the obstacle, $v_{B}$, to each velocity in $\mathrm{CC}_{\mathrm{A}, \mathrm{B}}$ thereby forming the Velocity Obstacle, VO, where $V O=C C_{A, B} \otimes v_{B}$ and $\otimes$ is the Minkowski vector sum operator. The VO then is the set of colliding absolute velocities between $\hat{A}$ and $\hat{B}$. By finding the union of the individual velocity obstacles, $V O=\bigcup_{i=1}^{m} V O_{B_{l}}$, where $\mathrm{m}$ is the number of obstacles, multiple obstacles can be avoided using a combined velocity obstacle. Therefore the robot can avoid a collision by selecting any velocity outside of the combined velocity obstacle; these velocities are known as aroidance velocities.

The dynamics of the vehicle are considered by computing the set of velocities that the vehicle can reach in the set time interval and are known as reachable velocities.
By choosing avoidance velocities that are also reachable velocities a set of reachable avoidance velocities is formed. These reachable avoidance velocities can then be searched using a cost function in order to select the velocity that best fulfils a set of heuristics.

Recently some modifications have been suggested to the Velocity Obstacle method. One modification is the introduction of a short time horizon so that priority is given to avoiding obstacles that are directed towards an imminent collision [7]. Another modification has been to create a nonlinear velocity obstacle capable of avoiding collisions with obstacles that do not move atong a linear path [8]. Finally, modifications to the Velocity Obstacle method, brought by the algorithm being proposed by this paper, the Time Varying Dynamic Window Algorithm, are explained in section that follows.

\section{TRME VARYTNG DYNAMIC WINDOW ALGORITHM}

The Time-Varying Dynamic Window algorithm, as suggested by the name, is a modification of the Dynamic Window algorithm with the inclusion of the Velocity Obstacle algorithm that is used to determine the admissible velocities. This approach has the benefit of using the wellstructured steps of the Dynamic Window approach and the ability to operate in a time-varying environment provided by the Velocity Obstacle Approach. The. need for this modification has also been found earlier, though independently, in [9], though this method fails to find the need for gradient velocity obstacles.

Similarly to the Velocity Obstacle Approach this method operates in Cartesian velocities rather than translational and angular velocity pairs. This decision was made due to the test vehicle platform - Cycab [10 - 11], using the IBEO ladar sensor [2] that provides velocity information on obstacles in Cartesian pairs.

In addition to the integration of these two methods a number of other improvements were made by the proposed Time Varying Dynamic Window Algorithm, namely: the holonomic constraints of a car-like vehicle were incorporated; the velocity obstacle was discretized for improved performance and converted to a gradient; and a new cost function was created.

\section{A. Holonomic Constraints}

Most methods developed for obstacle avoidance have been designed to be implemented on a holonomic robot with differential drive. Our method was required to be implemented on a car-like platform that required the consideration of non-holonomic constraints such as the inability to turn without forward motion (i.e. turning on the spot is not possible by the vehicle platform used in experiments - Fig 4).
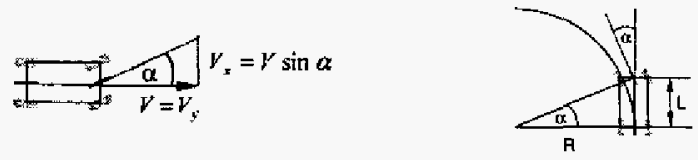

Fig. 3 Simplification of Vehicle to Bicycle 


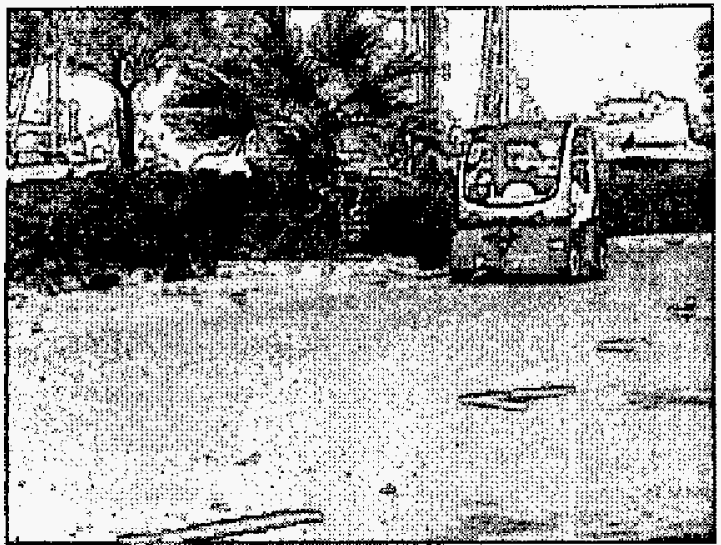

Fig. 4 Experimental Vehicle

The non-holonomic constraints of the vehicle were incorporated into the achievable velocitics step of the dynamic window approach structure by limiting the maximum horizontal velocity, $V_{x}$, based on the lateral velocity, $V_{Y}$. By either limiting the maximum curvature of the vehicle or by finding its rotational velocity by simplifying the vehictes motion to a two-wheel bicycle, Fig. 3,

$C=\frac{1}{R}=\frac{\tan \alpha}{L}=\frac{d \beta}{d s}$

the maximum steering angle can be calculated which is then used to limit the Cartesian velocity pairs based on the lateral velocity of the vehicle.

\section{B. Gradient Velocity Obstacle}

For the Velocity Obstacle method to interact with the Dynamic Window stage of the Dynamic Window Approach the Velocity Obstacle had to be converted from an absolute indicator of a collision to a gradient. The reason for this is when the Cost Function is used to select a velocity from the dynamic window it is possible that the whole dynamic window can contain collision velocities. In the case where an absolute velocity obstacle is used, the vehicle will no longer try to avoid a collision and will favour the other heuristics, in this case, speed and goal heading. By changing the velocity obstacle to a gradient, the vehicle can select velocities that will eventually place the vehicle along a safe path to avoid the obstacle.

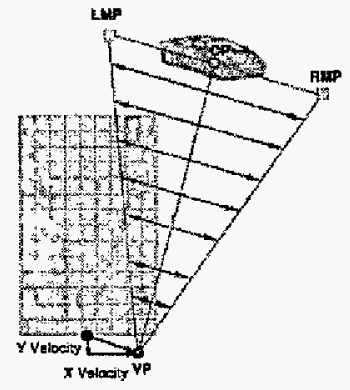

Fig. 5 Gradient Velocity Obstacles

Gradient velocity obstacles are implemented using line equations. The direction of the gradient is based on the slope of the line from the left-most point (LMP) to the rightmost point (RMP) of the obstacle. In order to find the gradient value it is necessary to find the line equations from the vehicle point (VP) along VP-RMP, VP-LMP, and VP$\mathrm{CP}$. The point $\mathrm{CP}$ is the point that is centred between LMP and RMP along the line LMP-RMP.

The velocity value of the current index is checked to lie within the limits set by the VP-LMP and VP-RMP lines then the distance from the related velocities of the current index to the PC-VP line and the total distance from the CPLMP / CP-RMP line both measured along the slope of the gradient are found using the equation:

$$
\text { gradientValue }=1-\frac{\text { distance }}{2 \times \text { totalDistance }}
$$

This then results in a gradient with a maximum value of 1 at the centre of the Velocity Obstacie and a value of 0.5 at its edge.

\section{Cost Function}

Similar to the cost function used in the Dynamic Window approach a function composed of three heuristics was used in this method to search for the next velocity of the vehicle. The three heuristics used were $\operatorname{angle}\left(V_{x} V_{y}\right)$, $\operatorname{speed}\left(V_{x}, V_{y}\right)$, and inhibition $\left(V_{x}, V_{y}\right)$.

The angle heuristic is used to provide the vehicle with a goal-directed behaviour by finding the heading of the vehicle if it takes the current $\left(V_{x} V_{y}\right)$ velocity pair and then subtracting this from the goal heading and normalizing this value with $\pi$. This results in a value of 1 if the velocity pair is directly towards the goal, and a value of 0 if in the totally opposite direction.

angle $\left(V_{x}, V_{y}\right)=\frac{\pi-\left[\tan ^{-1}\left(\frac{y \text { Goal }}{x \text { Goal }}\right)-\tan ^{-1}\left(\frac{V_{x}}{V_{y}}\right)\right]}{\pi}$

The speed heuristic makes the vehicle favour moving at faster velocities. The function for doing this is to normalize the current $\mathrm{V}_{\mathrm{y}}$ velocity by the fastest velocity of the vehicle, $V_{y \max }$.

$\operatorname{speed}\left(V_{x}, V_{y}\right)=\frac{V_{y}}{V_{y \max }}$

The inhibition heuristic converts the gradient of the velocity obstacles into a value that indicates the safety in choosing the velocity pair.

inhibition $\left(V_{x}, V_{y}\right)=1-$ window $\left(\right.$ index $_{V_{x}}$, index $\left._{V_{y}}\right)$

Therefore if the dynamic window value (represented by window $(x, y))$ is 0 , indicating the velocity will not cause a collision, the inhibition will be 1 and therefore maximized. However if the velocity is a collision velocity, the gradient at the point of the velocity pair will be subtracted from 1 making the velocity pair less likely to be chosen. This heuristic, together with the gradient of the Velocity Obstacles, ensures that the vehicle will head in a direction that avoids a collision.

Combining these three heuristics the Cost Function becomes:

$$
\begin{aligned}
C\left(V_{x}, V_{y}\right)= & \alpha \times \operatorname{angle}\left(V_{x}, V_{y}\right) \\
& +\beta \times \operatorname{speed}\left(V_{x}, V_{y}\right) \\
& +\chi \times \operatorname{inhhibition}\left(V_{x}, V_{y}\right)
\end{aligned}
$$


where $\alpha, \beta$ and $\chi$ are the cost function parameters of the angle, speed and inhibition function respectively.

\section{EXPERIMENTAL RESULTS}

The algorithm was tested on our test vehicle platform, which is a car-like vehicle operated using a $3 \mathrm{GHz}$ Pentium 4 erubedded computer, a second $\mathrm{PC}$ running a real-time linux kernel and two MPC555 microcontrollers. The algorithm was implemented on the embedded computer that is used for high-level behaviors, while the secondary PC and the MPC555 controllers are used for low-level behaviour consisting mostly of motor control.

For the purposes of obstacle avoidance, the test vehicle platform was equipped with an IBEO Ladar Sensor [2] that performs scans at a frequency of $10 \mathrm{~Hz}$ over a $270^{\circ}$ area (though only $180^{\circ}$ was utilized) at $0.25^{\circ}$ degree intervals. The sensor can track 25 objects internally and transmits up to 20 objects details via the CAN bus that lie within a set object output area normally of size $6 \times 6 \mathrm{~m}$. The range of the ladar sensor is dependent on reflectivity but ranges from $100 \mathrm{~m}$ at $90 \%$ reflectivity to $50 \mathrm{~m}$ at $10 \%$ reflectivity. Testing of the method was performed in a simulation environment and on our experimental test platform. For simulation, a model of the ladar sensor was used to provide data. The ladar sensor data was simulated over a $180^{\circ}$ field of view with any obstacle within a $20 \times 20 \mathrm{~m}$ radius becoming visible. In accordance with the ladar sensors data the left-most, closest, and right-most points of the obstacle were then provided.

The aim of testing was to create simulated environments that would occur on a typical inner-city road. Obstacles were modelled as circular figures, and by using different sizes were used to represent various moving obstacles such as pedestrians, cyclists, automobiles, and buses. Each obstacle was then given a starting point and a set velocity. Combinations of these obstacles were then used in the simulation of six possible scenarios. These scenarios were a static obstacle on collision path with the vehicle, a static obstacle on a non-collision path with the vehicle, an intersection scenario, a head on collision scenario, a lanemerge scenario and a sidewalk/adjacent lane scenario.

All tests were performed using the cost function parameters of $\alpha=0.3, \beta=0.1, \chi=0.6$ with the simulated vehicle operating at $7 \mathrm{~m} / \mathrm{s}(25 \mathrm{~km} / \mathrm{h})$, and obstacles operated up to a maximum of $16.5 \mathrm{~m} / \mathrm{s}(60 \mathrm{~km} / \mathrm{h})$. Of the total 248 test cases performed the algorithm had an $89.1 \%$ success rate at avoiding all obstacles while maintaining a safety distance of a meter, and of reaching the goal. The exclusion of cases with obstacles operating at $16.5 \mathrm{~m} / \mathrm{s}$ raised this to $97.2 \%$ indicating that the current sensor setup is not yet adequate for operating with obstacles moving at velocities greater than $7 \mathrm{~m} / \mathrm{s}$.

In Figure 6 an example test of the simulation environment can be seen in which three obstacles are present. All of the obstacles and the vehicle are moving at $7 \mathrm{~m} / \mathrm{s}$. Each instance of the vehicles and obstacles represents a critical stage of the simulation. The first stage shows the initial position of all the obstacles and the vehicle prior to the simulation starting. In the second stage shows when the vehicle has just detected the first obstacle and is about to begin an evasive maneuver to the left. By the third stage the vehicle has detected obstacle two and using the combination gradient velocity obstacle continues to avoid to the left. The fourth stage shows the vehicle detecting the third obstacle and the gradient velocity obstacle of the third obstacle causes the vehicle to reduce its speed to avoid the collision. The fifth stage shows the vehicle maintaining a slow admissible velocity about to avoid the thitd obstacle, then increase speed and reach the goal.

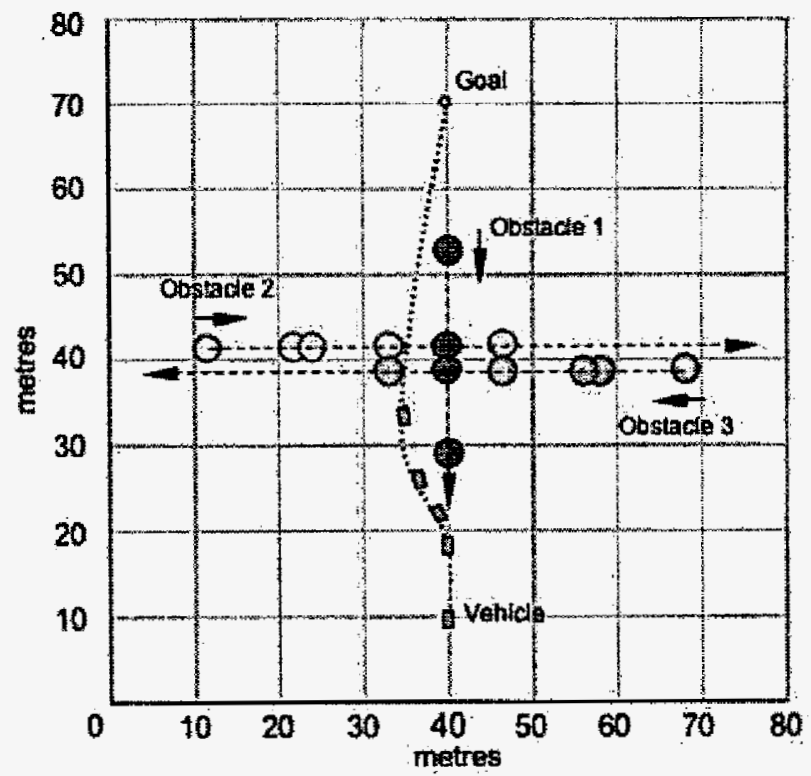

Fig. 6 Intersection Scenario Test in Simulation Environment

In addition to simulation, testing was also performed on the test vehicle platform. Initial tests were performed at low speeds $(0.5 \mathrm{~m} / \mathrm{s})$ with static and slow moving obstacles, Fig 7. The results were promising and gave initial success.

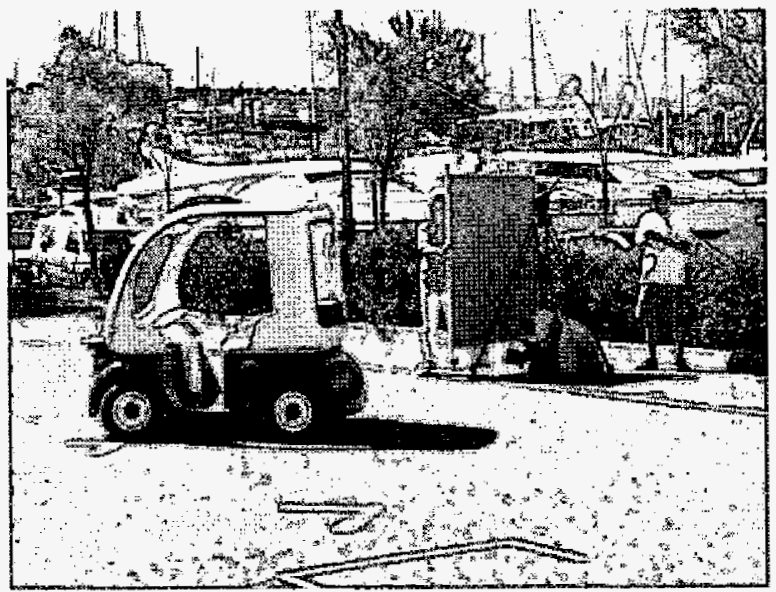

Fig. 7 Test site

During simulation testing the cause of collisions was found to be local minima occurring in the gradient velocity obstacles upon the combination of multiple velocity obstacles. In the future calculating the gradient velocity obstacle after all velocity obstacles have been combined will extend this method. This would also allow the algorithm to 
follow a slow moving obstacle or stop to avoid a collision thereby giving success in the abortive manoeuvre of a similar method [9].

\section{CONCLUDING REMARKS}

This paper has presented an algorithm that is capable of avoiding a collision in a time-varying environment at high speeds. Future work may include further testing under a variety of the city road conditions as well as fine tunings, if required, of the developed solutions.

The entirety of the performed in-field testings (which included not just experimenting with dynamic obstacles, typical for city roads, but also undertaking an unsignalised intersection cooperative traversal, a cooperative overtaking manoeuvre, a manoeuvre requiring the vehicles to drive one behind each other while maintaining distance and track control and finally a simple road-lane following driving manoeuvie) demonstrates that driverless vehicles operating along side human drivers will hopefully be a not too distant reality.

With the potential to improve urban mobility in a number of sustainable ways, cooperative driverless vehicles will be seen in: (i) resorts and coastal (close-to-beach) areas; (ii) retirement villages; and (iii) existing suburbs and new residential areas where they could provide a door-to-door on-call service, designed to complement existing public transport means, by providing an efficient shuttle service between defined pick-up and drop off points (schools, bus and railway stations) and thus complementing the existing transport system and optimising traffic flows as they pass.

\section{REFERENCES}

[1] User Needs Analysis and Analysis of Key Technologies: Report on Existing Technologies for vehicles. Retrieved 07 Feb. 2005, from bttp: $/$ www.cybercars.org/docs/D 1 Part1-UserNeed.doc

[2] IBEO LD Aufomotive. Retrieved 07 Feb. 2005, from http//www.ibeoas.de/html/prod/prod_ld_autom.htmlT.

[3] J Kolodko and L Vlacic. Motion Vision: Design of Motion Compact Sensing Solutions for Navigation of Autonomous Systems, The IEE Publishers, 2004

[4] Y. Hwang, and N. Ahuja, Gross Motion Planning - A Survey. $A C M$ Computing Surveys, vol. 24 (3), 1992, pp. 219-291.

[5] B. Kohout, Challenges in Real Time Obstacle Avoidance, AAAI Spring Symposium on Real-Time Autonomous Systems, 2000, California, USA

[6] D. Fox, W. Burgard, and S. Thrun, The Dynamic Window Approach to Collision Avoidance, IEEE Robotics and Automation Magazine, vol 4 (1), 1997, pp. 23-33.

[7] P. Fiorini and Z. Shiller, Motion planning in Dynamic Environments using Velocity Obstacles, Int. Joumal on Robotics Research, vol 17 (7), 1998, pp. 711-727.

[8] Z. Shiller, F.Large and S. Sekhavat, Motion Planning in Dynamic Environments: Obstacles Moving Along Arbitary Trajectories, Int. Conf. On Robotics and Automation, 2001, Seoul, Korea.

[9] D. Castro, U. Nunes and A. Ruano, Reactive Local Navigation, Proceedings of the .28th Industrial Electronics Society Conference, 2002, Sevilla, Spain.

[10]J. Baber, J Kolodko, L Vlacic, T Noel and M Parenl. Cooperative Autonomous Driving in Cities, To appear in IEEE - $\mathbf{R} \&$ A Magazine, March 2005.

[11] J Kolodko, L Vlacic, Cooperative Autonomous Driving at the Intelligent Control Systems Laboratory, IEEE Intelligent Systems: Putting A I into Practice, Vol. 18, No.4, 2003, pp:8-11 